\title{
The growing role of professional societies in educating clinicians in genomics
}

\author{
Teri A. Manolio, MD, $\mathrm{PhD}^{1}$ and Michael F. Murray, $\mathrm{MD}^{2}$; for the Inter-Society Coordinating Committee \\ for Practitioner Education in Genomics
}

Recent identification of numerous genetic variants influencing metabolism of commonly used drugs, responses to chemotherapy, and risk of familial and sporadic diseases, coupled with improved technologies for detecting these variants at continually declining costs, have opened exciting prospects for improving clinical care through genomic applications. ${ }^{1}$ Lack of education in genomics among physicians and allied health personnel not only delays appropriate clinical application of these promising approaches but also could lead to erroneous uses with serious consequences. ${ }^{2}$ To address these challenges and keep pace with expanding applications of genomics in fields such as drug selection, cancer treatment, high-risk screening, and undiagnosed diseases, several physician professional societies joined the National Human Genome Research Institute in a 2-day meeting in January 2013 to discuss the growing opportunities for and barriers to educating physicians in genomics. As conceived at that meeting, these and other professional societies engaged with the National Human Genome Research Institute and each other in February 2013 to form an Inter-Society Coordinating Committee on Practitioner Education in Genomics (ISCC, see http://www.genome. gov/27552294). Here, we describe those discussions and outline the ISCC's plans for uniting professional societies to facilitate clinician education. Membership is open to any health professional society involved in educating health-care practitioners in applying genomic results to clinical care and willing to contribute to ISCC efforts. All such societies are enthusiastically invited to convey their interest in joining by contacting the authors.

\section{OPPORTUNITIES FOR PHYSICIAN EDUCATION IN GENOMICS}

Most physicians feel poorly prepared to utilize genomic advances in drug therapy, cancer treatment, and risk of familial and sporadic diseases in addition to being overwhelmed by the mass of information many feel they are expected to master. ${ }^{3}$ Such concerns may have been fueled as much by proclamations of a "genomics revolution"4 as by the recent explosion of findings relating the genome to disease. ${ }^{5}$

In fact, a relatively small (but growing) number of genomic findings is ready for direct clinical application at present, and findings that are ready typically represent incremental advances on current clinical practices-more of an evolution than a revolution. Assessing a pharmacogenetic variant along with creatinine clearance in drug dosing, for example, or using tumor variants as well as histology in predicting treatment response, can be viewed as expansions of the laboratory-based armamentarium already available to clinicians. In contrast, direct-to-consumer marketing of genomic tests raises quite different challenges, including the daunting proposition of a physician being handed a patient-initiated genome-wide scan and expected to provide an expert interpretation. ${ }^{6}$

Surveys of physicians to date have indicated that many feel unprepared to order and interpret genomic tests and wish to improve their knowledge, but most feel able to commit only a few hours to such education. Surveys of current knowledge and practice suggest substantial error rates in physicians' orders for genetic tests, ${ }^{2,7}$ as well as frequent and unnecessary duplication of tests. Nascent systems for standardized reporting of results and incorporation into interoperable but secure electronic medical records will be critical to improving confidence and competence in working with genomic findings. Such successes can be used to encourage additional learning as knowledge and experience accrue.

Although physicians are not unique in their need for genomics education, they have been somewhat slow to embrace it. ${ }^{8}$ Many allied health professionals have been more active in genomics education. ${ }^{9}$ Speed in adoption also varies across nongenetic medical specialties, with fields such as oncology and pathology at the leading edges of implementation. Such variations provide opportunities for sharing efforts of early adopters across professions and specialties, making intersociety coordination even more compelling.

\section{CHALLENGES AND BARRIERS TO PHYSICIAN GENOMICS EDUCATION}

Unique aspects of postgraduate physician education include the strongly patient-centric, case-based approach to learning. The almost insurmountable time and informational pressures under which physicians work mandate a highly focused, practical approach to presenting new knowledge. Tools such as point-of-care computerized decision support are beginning 
to be developed and hold promise for directing physicians to the information they need precisely when they need it. ${ }^{10}$ Credentialing requirements for ordering sophisticated tests such as genome sequencing could be powerful incentives for education, though at the cost of restricting use. Inclusion of genomic information in specialty board examinations and maintenance of certification testing may provide another unique and powerful motivator for physician education; indeed, given their many other certification requirements, inclusion in specialty boards is likely a must for genomics to get any attention at all.

The rapid evolution of genomic understanding risks leaving the typical nonspecialist physician with knowledge that quickly becomes outdated, such as the continued perception that genomic science is mainly relevant to rare, esoteric conditions. Medical genetics is viewed as dealing largely with these exotic, often early-onset conditions, with little opportunity for focused training in subspecialty-specific applications. The complexities of consent and counseling related to genomic testing, often with reproductive or family implications, are daunting to most clinicians and complicated by the limited availability of genetic counselors. Further barriers including the rapid pace of technologic and informatic innovations, evolving accuracy of the testing, complexity of genomic concepts, and obscure jargon in even basic presentations can frustrate the most concerted attempts to educate oneself in this field.

\section{OPPORTUNITIES FOR COLLABORATIONS IN EDUCATIONAL EFFORTS}

The confluence of readily applicable genomic findings, heightened interest, commonality of needs across specialties, and availability of educational materials from early adopter groups (such as the case studies provided at http://www.g-3-c.org/en/) make collaboration across societies timely and opportune. The National Human Genome Research Institute has thus worked with several professional societies and other National Institutes of Health institutes to identify needs and opportunities for practitioner education in applying genomic results to clinical care. The societies strongly urged establishment of the ISCC to facilitate their interactions and enhance their effectiveness in educating their memberships. The group has agreed to focus initially on physicians, who have lagged considerably behind other health professionals in embracing genomics, but hopes to soon engage the many allied practitioner groups vital to effective patient care. Key goals, proposed activities, and metrics of success have been outlined (see Supplementary Tables S1 and S2 online).

The ISCC recognizes the primacy of individual societies and specialty boards in defining practice guidelines and certification requirements for their members. Its role is thus to support and stimulate the activities of these societies but not to interfere with their internal processes or their role in guiding their members toward the effective practice of genomic medicine. As noted above, all health professional societies willing to contribute to ISCC efforts are enthusiastically invited to join.

\section{CONCLUSIONS}

Sufficient numbers of genomic findings, largely in pharmacogenomics and oncology as well as single-gene disorders such as arrhythmias and cardiomyopathies, have now been identified to support effective educational efforts in the clinical application of such variants. The ISCC proposes to use this initial corpus as a base for societies to introduce their members to genomic medicine applications. Educational efforts begun now will enable practitioners to act on the currently modest number of variants relevant to a given patient and context and to respond effectively to the growing opportunities for applying genomic results in clinical care.

\section{SUPPLEMENTARY MATERIAL}

Supplementary material is linked to the online version of the paper at http://www.nature.com/gim

\section{ACKNOWLEDGMENTS}

This commentary builds on work from the National Human Genome Research Institute summit on 25 January 2013, regarding needs of and interactions among professional societies for educating physicians in genomics and announces the formation of an Inter-Society Coordinating Committee for Practitioner Education in Genomics to promote genomic medicine education.

\section{DISCLOSURE}

The authors declare no conflict of interest.

\section{REFERENCES}

1. MCCarthy JJ, McLeod HL, Ginsburg GS. Genomic medicine: a decade of successes, challenges, and opportunities. Sci Trans/ Med 2013;5:189sr4.

2. Brierley KL, Blouch E, Cogswell W, et al. Adverse events in cancer genetic testing: medical, ethical, legal, and financial implications. Cancer J 2012;18:303-9.

3. Selkirk CG, Weissman SM, Anderson A, Hulick PJ. Physicians' preparedness for integration of genomic and pharmacogenetic testing into practice within a major healthcare system. Genet Test Mol Biomarkers 2013;17:219-25.

4. Zuger A. Genomics as a final frontier, or just a way station. http://www.nytimes. com/2012/02/28/health/views/genomics-as-a-final-frontier-or-just-a-waystation.html?_r=0. Accessed 18 June 2013.

5. Hindorff LA, MacArthur J, Morales J, Junkins HA, Hall PN, Klemm AK, and Manolio TA. A Catalog of Published Genome-Wide Association Studies. www.genome.gov/gwastudies. Accessed 19 June 013.

6. Giovanni MA, Fickie MR, Lehmann LS, et al. Health-care referrals from direct-toconsumer genetic testing. Genet Test Mol Biomarkers 2010;14:817-9.

7. Plon SE, Cooper HP, Parks B, et al. Genetic testing and cancer risk management recommendations by physicians for at-risk relatives. Genet Med 2011;13: 148-54.

8. Feero WG, Green ED. Genomics education for health care professionals in the $21^{\text {st }}$ century. JAMA 2011;306:989-90.

9. Calzone KA, Jerome-D'Emilia B, Jenkins J, et al. Establishment of the genetid genomic competency center for education. J Nurs Scholarsh 2011;43:351-8.

10. Hoffman MA, Williams MS. Electronic medical records and personalized medicine. Hum Genet 2011;130:33-9. 